\title{
Re: The Agency for Healthcare Research and Quality (AHRQ) Practice-based Research Network (PBRN) Relationship: Delivering on an Opportunity, Challenges, and Future Directions
}

\author{
David Meyers, MD and Mike Parchman, MD (J Am Board Fam Med 2011;24:493.)
}

We read with great interest the commentary by Pace and West in this issue of the Journal. We believe that their willingness to share experiencebased criticism of AHRQ's PBRN initiative and our PBRN Master Contract program is strong evidence of the healthy partnership that has developed between AHRQ and the PBRN community at large, and the 10 master contractors in specific. We are committed to ensuring that this partnership continues to mature and deepen and recognize that listening is one of the best ways to strengthen the AHRQ PBRN initiative.

In 2007, AHRQ expanded its PBRN initiative to include a new mechanism by which it could fund rapid-cycle research and implementation projects to advance its mission of improving the health and health care of all Americans. The PBRN Contract partnership was a 5-year project involving 10 PBRN partners. Through this initiative AHRQ issued 24 requests for task orders and awarded $\$ 17.5$ million through 38 individual task orders to the 10 PBRN Master Contractors. With this funding they conducted observational studies of primary care practices, field tested evidence-based tools in real-world settings, and conducted dissemination and implementation research. A few of the topics covered include: the use of health IT to

\footnotetext{
This article was externally peer reviewed.

Submitted 6 July 2011; revised 6 July 2011; accepted 6 July 2011.

From the Center for Primary Care, Prevention, and Clinical Partnerships, Director, AHRQ PBRN Initiative.

Funding: none.

Conflict of interest: none declared.

Corresponding author: David Meyers, Center for Primary Care, Prevention, and Clinical Partnerships, Director, AHRQ PBRN Initiative (E-mail: David.Meyers@ahrq.hhs.gov).
}

improve the quality of care transitions, estimating the direct and indirect costs to the primary care practice of quality measurement data collection and reporting, management of community-acquired MRSA infections, and reducing inappropriate use of antibiotics. This project has allowed AHRQ and its federal partners to obtain timely answers to relevant questions across the diversity of small and medium sized primary care offices that form the core of America's primary health care delivery system.

Elsewhere in this issue, we give tribute to the tremendous contributions of Dr. David Lanier to AHRQ's PBRN initiative. Early in 2011 the AHRQ PBRN initiative was without senior leadership. This may have contributed to some of the frustrations Dr. Pace and West experienced. This spring, Dr Michael Parchman joined AHRQ as the new director of our PBRN initiative. He brings with him the experience of a seasoned PBRN director. The PBRN community can be assured that their voice and concerns will continue to be heard at AHRQ. We share many of the concerns expressed by Dr. Pace and West and understand the potential value of their suggestions. We have been and will continue to listen to the research and practice communities as we invest in the transformation of the primary care health system. We believe that our national aim of a health system that delivers better care, more affordable care, and more patient centered care can only be achieved on the foundation of a revitalized primary care system. And that primary care PBRNs are living laboratories where innovations will be discovered, refined, and disseminated. 